\title{
ANALYTIC FUNCTIONS IN THE IRREGULAR FIELD OF ALL NUMERICAL FUNCTIONS*
}

BY

\author{
E. T. BELL
}

1. Introduction. To distinguish them from the elements with which we shall be chiefly concerned, we shall call real or complex numbers scalars. As it is usually immaterial in the sequel whether the scalars are real or imaginary, either may be understood unless the contrary is expressed. Scalars will be denoted by small Latin letters, $a, b, \cdots, x, y, \cdots$.

For the moment only, let $\alpha, \beta, \cdots, \xi, \eta, \omega$ denote elements of an abstract field $F$ whose zero, unit elements are $\omega, \eta$ respectively, and in which sums, products, quotients, differences are written as usual, $\alpha+\beta, \alpha \beta, \alpha / \beta$, $\alpha-\beta$. The properties of products such as $a \alpha$ are assumed in the customary form. $\dagger$ To the classical postulates and definitions of $F$, it is convenient for our purpose to add the following definition: An element $\xi$ of $F$ is called regular or irregular according as there exists or does not exist a uniquely defined element $\xi^{\prime}$ in $F$, called the reciprocal of $\xi$, such that $\xi \xi^{\prime}=\eta$. The unique irregular element in $F$ is $\omega$.

Suppose now that elements $\alpha, \beta, \cdots$ and the four fundamental operations upon them (addition, subtraction, multiplication, division) are defined so that the resulting system $I F$ differs from $F$ only in the existence in $I F$ of an infinity of irregular elements. Then $I F$ is called an irregular field. The postulates for $I F$ were precisely stated in a former paper, $\ddagger$ and numerous instances were given there and elsewhere. $\S$ The foregoing description of $I F$ is sufficient for the present, as we shall discuss an instance of $I F$ in some detail. In the papers cited the algebra (theory of finite processes) in $I F$ was fully treated; here, we consider the analysis (theory of essentially infinite processes) so far as it relates to functions in $I F$ having power series expansions in $I F$, for the particular instance mentioned. Elements of the $I F$ considered will be denoted by small Greek letters, $\alpha, \beta, \cdots, \omega, \eta, \xi, \cdots$.

Before proceeding, it may be of interest to state an example of the similarities and differences between analytic functions in a scalar field and

\footnotetext{
* Presented to the Society, April 5, 1930; received by the editors in February, 1930.

† See, for example, L. E. Dickson, Algebren und ihre Zahlentheorie, 1927, pp. 23-24.

$\ddagger$ E. T. Bell, Annals of Mathematics, vol. 27 (1926), p. 511.

$\S$ E. T. Bell, Algebraic Arithmetic, 1927.
} 
their correspondents in $I F$. It was shown by Tannery* that if $S(x), C(x)$ are uniquely defined for all values of $x$, and have the addition theorems

$$
\begin{aligned}
& S(x+y)=S(x) C(y)+C(x) S(y), \\
& C(x+y)=C(x) C(y)-S(x) S(y),
\end{aligned}
$$

and if there exists a single value of $x$ for which $C(x), S(x)$ are continuous, then they are continuous for all $x$, and, the trivial case where both vanish identically being excluded,

$$
S(x)=e^{a x} \sin b x, \quad C(x)=e^{a x} \cos b x,
$$

where $a, b$ are constants. If in addition

$$
S^{2}(x)+C^{2}(x)=1,
$$

then $S(x)=\sin b x, C(x)=\cos b x$. Further instances of completely solved systems of functional equations will be found in Osgood (loc. cit.) and in Van Vleck and H'Doubler. $\dagger$ Remarks similar to the following, for the above set, apply to all.

We note first that the values of $S(x), C(x)$ are scalars, as also is $x$. We shall require our functions in $I F$, that is their "values," to be in the same $I F$ as their arguments. Second, if $x$ is not scalar, continuity is not significant, and hence must be dropped in $I F$. Third, the right-hand member of (1.3) is the unit scalar; in $I F$ it must be replaced by $\eta$, the unit element in $I F$, which is such that, if $\xi$ is any element of $I F, \eta \xi=\xi$. Fourth, the (scalar) solution of (1.1), (1.3) having been shown to exist under the stated hypotheses, the power series for the sine and cosine follow in the usual way, and are absolutely convergent for all finite values of the argument.

In $I F$ the situation corresponding to (1.1), (1.3) is

$$
\begin{aligned}
& S(\alpha+\beta)=S(\alpha) C(\beta)+C(\alpha) S(\beta), \\
& C(\alpha+\beta)=C(\alpha) C(\beta)-S(\alpha) S(\beta),
\end{aligned}
$$

where $S(\xi), C(\xi)$ are to be uniquely defined for every $\xi$ in $I F$; and

$$
S^{2}(\xi)+C^{2}(\xi)=\eta .
$$

The conclusion is that

$$
S(\xi)=\sum_{r=0}^{\infty} \frac{(-1)^{r} \xi^{2 r+1}}{(2 r+1) !}, \quad C(\xi)=\sum_{r=0}^{\infty} \frac{(-1)^{r} \xi^{2 r}}{(2 r) !} ;
$$

* See, for example, W. F. Osgood, Lehrbuch der Funktionentheorie, 1926, pp. 582-586.

† E. B. Van Vleck and F. H' Doubler, these Transactions, vol. 17 (1916), pp. 9-49. 


$$
\begin{aligned}
S(-\xi) & =-S(\xi), & C(-\xi) & =C(\xi) ; \\
S(\omega) & =\omega, & C(\omega) & =\eta,
\end{aligned}
$$

where $\omega$ is the (unique) zero element in $I F$, defined by $\omega \xi=\omega$ for all $\xi$ in $I F$ ( $\omega$ also is irregular).

Further, from the definition of derivatives in $I F$ with respect to $\xi$, indicated by $D_{\xi}$, it follows that $D_{\xi} S(\xi)=C(\xi), D_{\xi} C(\xi)=-S(\xi)$. The next is in striking contrast to the scalars $S(x), C(x)$. In the instance of $I F$ considered, there is uniquely determined a constant finite scalar, say $p$, when $\xi$ is a designated element of $I F$, such that $S(\xi), C(\xi)$ as in (1.4) are reducible to the forms

$$
\begin{aligned}
& S(\xi)=C^{\prime}(\xi) \sin p+S^{\prime}(\xi) \cos p, \\
& C(\xi)=C^{\prime}(\xi) \cos p-S^{\prime}(\xi) \sin p,
\end{aligned}
$$

where $C^{\prime}(\xi), S^{\prime}(\xi)$ are certain finite linear homogeneous functions of certain elements of $I F$ which are uniquely determined when $\xi$ is given, and which further satisfy the same functional equations (1.11), (1.31), (1.5) as $C(\xi)$, $S(\xi)$. The sense in which infinite processes exist, or are convergent, in $I F$ will appear as we proceed.

The similarities and differences illustrated above arise from two sources: the existence in $I F$ of an infinity of irregular elements, and the specific interpretations assigned to the four fundamental operations.

2. The $I F$ of all numerical functions. We shall recall from previous papers a minimum of definitions and theorems to make the present selfcontained.

If for all finite integral values $>0$ of the scalar variable $x$ the scalar function $\xi(x)$ is finite and uniform, $\xi(x)$ is called a numerical function of $x$, and is said to be regular or irregular according as $\xi(1) \neq 0$ or $\xi(1)=0$. The unit, zero numerical functions, $\eta(x), \omega(x)$ are defined by

$$
\eta(1)=1, \eta(n+1)=0, \omega(n)=0 \quad(n=1,2, \cdots) .
$$

The numerical functions $\alpha(x), \beta(x)$ are defined to be equal when and only when $\alpha(n)=\beta(n)$ for all integers $n>0$. If $\alpha(x), \beta(x)$ are equal, the equality is written $\alpha=\beta$. Hence the following are equivalent statements:

$$
\alpha=\beta, \alpha(n)=\beta(n) \quad(n=1,2, \cdots) .
$$

As such symbols $\alpha, \beta, \cdots$ occur only in relations of equality as just defined, it is unnecessary to define them separately. The special irregular field $I F$ to be discussed has as its elements the set of all $\alpha, \beta, \cdots, \eta, \omega, \xi, \cdots$, where $\alpha(x), \beta(x), \cdots, \xi(x), \cdots$ is the set of all numerical functions; $\eta(x), \omega(x)$ are as above defined, and $\xi$ is regular or irregular according as $\xi(x)$ is regular or 
irregular. To avoid repeating $n=1,2, \cdots$, we make the convention that any relation involving $n$ is to hold for all integral values $>0$ of $n$. The fundamental operations in $I F$ are defined by the following pairs of statements, postulated to be equivalent:

$$
\begin{array}{ll}
\phi=c \xi, & \phi(n)=c \xi(n) ; \\
\phi=\alpha+\beta, & \phi(n)=\alpha(n)+\beta(n) ; \\
\phi=\alpha \beta, & \phi(n)=\sum \alpha(d) \beta(t),
\end{array}
$$

the summation referring to all distinct matrices $(d, t)$ of integers $d>0, t>0$ such that $d t=n$.

It was shown elsewhere* that if and only if $\xi$ is regular, there exists in IF a uniquely determined element $\xi^{\prime}$, called the reciprocal of $\xi$, such that $\xi \xi^{\prime}=\eta$, and an explicit form for $\xi^{\prime}(n)$ in terms of $\xi(d)$, where $d$ runs through the positive integral divisors of $n$, was given. Here it is sufficient to know that $\xi^{\prime}$ exists when and only when $\xi$ is regular. Writing $\xi^{\prime}=\eta / \xi=\xi^{-1}$ ( $\xi$ regular), we thus define division in $I F$.

From (2.2), (2.3), $a \alpha+b \beta+\cdots+c \gamma$ is defined, and from (2.4) the definition of $\xi_{1} \xi_{2} \cdots \xi_{r}$ follows:

$$
\phi=\xi_{1} \xi_{2} \cdots \xi_{r}, \quad \phi(n)=\sum \xi_{1}\left(n_{1}\right) \xi_{2}\left(n_{2}\right) \cdots \xi_{r}\left(n_{r}\right),
$$

the summation referring to all matrices $\left(n_{1}, n_{2}, \cdots, n_{r}\right)$ of integers $n_{j}>0$ $(j=1, \cdots, r)$ such that $n=n_{1} n_{2} \cdots n_{r}$. If $\xi_{1}=\xi_{2}=\cdots=\xi_{r}$, we write $\phi=\xi^{r}$. If $\xi$ is regular, and $\xi^{\prime}$ is the reciprocal of $\xi, \xi^{-r}$ is defined by $\xi^{-r}=\xi^{\prime r}$. If $\alpha$ is any element ( $\omega$ not excluded) of $I F$, by definition $\alpha^{0}=\eta$. If $\xi$ is any element of $I F, \eta \xi=\xi, \omega \xi=\omega$; the unit element $\eta$ is unique, as also is the zero element $\omega$. The properties of scalar multiplication defined in (2.2), addition in (2.3) and multiplication in (2.4) are abstractly identical with those of the similarly named operations in a field, and hence with respect to these operations the elements of $I F$ form a ring, say $I R$. Adjoining to $I R$ division in $I F$ as above defined, we see that $I F$ is abstractly identical with common algebra, except that in $I F$ division is impermissible by each of an infinity of elements of $I F$ (the irregular elements), instead of merely by the unique zero. Details, if desired, will be found in the papers cited; the foregoing is sufficient for what follows.

3. Components. Throughout this section, $\xi$ is an arbitrary element of $I F, r$ is an arbitrary integer $\geqq 0$, and $p=\xi(1)$. We shall define elements $\xi_{a}(a=0, \cdots, r)$ in $I F$, the components of $\xi^{r}$, such that

* E. T. Bell, Tôhoku Mathematical Journal, 1921. 


$$
\xi^{r}=r ! \sum_{a=0}^{r} \frac{p^{r-a}}{(r-a) !} \xi_{a} .
$$

It is easily shown that if the $c_{a}$ are scalars, and the $\xi_{a}^{\prime}$ elements of $I F$, a resolution of the form

$$
\xi^{r}=\sum_{a=0}^{r} c_{a} \xi_{a}^{\prime}
$$

is, if it exists, unique. Thus (3.1), when the $\xi_{a}$ are determined, will be the unique resolution of $\xi$ into its components in $I F$. If $s$ is an integer $<0$, and $\xi$ is irregular, $\xi^{s}$ does not exist; if $\xi$ is regular, $\xi^{s}=\left(\xi^{\prime}\right)^{-s},-s>0, \xi^{\prime}=$ the reciprocal (in $I F$ ) of $\xi$, so that this case is included in (3.1). Hence (3.1) is the general case. The resolution of powers into components enables us to pass, as will be seen later, directly from finite to infinite processes in $I F$. We proceed to find the components $\xi_{a}$.

Let $n=q_{1} t_{1} \cdots q_{j}{ }^{t_{j}}\left(t_{1}>0, \cdots, t_{j}>0\right)$ be the resolution of $n>1$ into a product of powers of distinct primes $q_{1}, \cdots, q_{t}$ (1 is not a prime); write $\pi(1)=0, \pi(n)=t_{1}+\cdots+t_{j}$. Then no resolution of the integer $m>0$ into more than $\pi(m)$ factors $>1$ is possible.

By definition, we take

$$
\begin{array}{rlrl}
\xi_{a}(n)=0 & (a>\pi(n), n=1,2, \cdots) ; \\
\xi_{0}=\xi^{0}=\eta ; \quad \xi_{b}=\omega & & (b<0) .
\end{array}
$$

Hence we need consider only $\xi_{a}(n), n>1,0<a \leqq \pi(n)$.

Let $n$ be an integer $>1$; let $a_{1}, a_{2}, \cdots, a_{s}$ be $s$ integers $>0$, and $P_{1}$, $P_{2}, \cdots, P_{s}$ be $s$ distinct integers $>1$ which, without loss of generality, may be assumed in ascending order, $P_{1}<P_{2}<\cdots<P_{s}$, such that

$$
n=P_{1}^{a_{1}} P_{2} a_{2} \ldots P_{s}^{a_{s}} .
$$

Write $a=a_{1}+a_{2}+\cdots+a_{s}$. We shall call (3.4) a decomposition of $n(>1)$ of degree $a$.

Consider $\xi^{r}(n)(n>1)$. If $b>r$, no decomposition of $n$ of degree $b$ contributes to $\xi^{r}(n)$. By the definition in $\S 2$ of multiplication in $I F$,

$$
\xi^{r}(n)=\sum \xi\left(n_{1}\right) \xi\left(n_{2}\right) \cdots \xi\left(n_{r}\right),
$$

$\sum$ having the signification there stated. Hence, if we define $\xi_{a}(n)$ to be the sum

$$
\sum \frac{\left(\xi\left(P_{1}\right)\right) a_{1}\left(\xi\left(P_{2}\right)\right)^{a_{2}} \cdots\left(\xi\left(P_{s}\right)\right)^{a_{s}}}{a_{1} ! a_{2} ! \cdots a_{s} !}
$$

extended over all decompositions of $n$ of degree $a$ when $a \leqq r$, the total contribution of all the decompositions of degree $a$ of $n$ to $\xi^{r}(n)$ is 


$$
\frac{r ! p^{r-a}}{(r-a) !} \xi_{a}(n),
$$

since, for example, the particular decomposition (3.4) must be written

$$
n=1^{r-a} P_{1} a_{1} P_{2}^{a_{2}} \cdots P_{a}^{a_{a}}
$$

in order to contribute to $\xi^{r}(n)$.

Finally, then, with $\xi_{a}(n)(a \geqq 0, n>0)$ as defined in (3.2), (3.3), (3.5), we see by these and (3.6) that the required resolution of $\xi^{r}(r \geqq 0)$ into its components $\xi_{a}$ is (3.1).

In discussing infinite processes in $I F$, all powers are resolved into their components. From the definitions, all products of components are products in $I F$. The last remark may be emphasized, as the final theorems derived from power series in $I F$ are of precisely the same forms as their correspondents in a scalar field.

In (3.1) we have resolved a power into its components. There is a reciprocal resolution of $\xi_{r}$ into a polynomial in $\xi$ of degree $r$. Writing (3.1) for the moment in the form

$$
\xi^{r}=\sum_{a=0}^{r} M_{a}(r) p^{r-a \xi_{a}},
$$

we observe that the $M_{a}(r)$ are rational numbers depending only upon $a, r$ and that the sequence $\xi_{0}, \xi_{1}, \ldots$ is uniquely determined in $I F$ when $\xi$ is given. In (3.7) replace $r$ by $0,1, \cdots, r$, and solve the resulting system of equations (of determinant 1) for the $\xi_{a}$. Then

$$
\xi_{r}=\sum_{a=0}^{r} N_{a}(r) \xi^{a},
$$

where the $N_{a}(r)$ are rational numbers depending only upon $a, r$, and the representation (3.8) is unique. The explicit forms of the $N_{a}(r)$ will not be required.

For completeness, we state the components of sums and products. Let $\alpha, \beta$ be any elements of $I F$. Write $\phi=\alpha+\beta, \psi=\alpha \beta$. We seek $\phi_{r}, \psi_{r}$, where $r$ is an integer $\geqq 0$. The results are

$$
(\alpha+\beta)_{r}=\sum_{a=0}^{r} \alpha_{a} \beta_{r-a},
$$

as may be proved directly from the definition of $\xi_{a}(n)$, or immediately from the exponential theorem as in $\$ 6$;

$$
\psi_{r}=(\alpha \beta)_{r}=\sum_{a=0}^{r} N_{a}(r) \alpha^{a} \beta^{a} ;
$$


and, therefore,

$$
(\alpha \beta)_{r}=\sum_{a=0}^{r} \sum_{b=0}^{a} \sum_{c=0}^{a} N_{a}(r) M_{b}(a) M_{c}(a) \alpha_{b} \beta_{c} .
$$

The following is a useful relation. Let $x$ be an arbitrary scalar, $\xi$ an arbitrary element of $I F$. Then

$$
(x \xi)_{r}=x^{r} \xi_{r} .
$$

4. Derivatives in $I F$. The following definitions enable us to attain complete isomorphism between derivatives of functions in $I F$ and the like for scalars. The derivatives $D_{\xi} \xi^{a}, D_{\xi} \xi_{a}$ of $\xi^{a}, \xi_{a}$, where $a$ is an integer $\sum_{0}$, and $\xi$ is regular, are the respective elements $a \xi^{a-1}, \xi_{a-1}$ of $I F$, - that is,

$$
D_{\xi} \xi^{a}=a \xi^{a-1}, D_{\xi} \xi_{a}=\xi_{a-1}(a \gtreqless 0) .
$$

If $\xi$ is irregular, (4.1) by definition is restricted to the case $a>0$. From (4.1) there follow easily all the formal properties of derivatives of sums, products, quotients, etc., in $I F$, in complete isomorphism with the like for scalars.

5. Analytic functions in $I F$. Let $\xi$ be an arbitrary element of $I F$, and let $R$ be the region of convergence of $f(x)$, the scalar function defined in $R$ by

$$
f(x)=\sum_{r=0}^{\infty} c_{r} x^{r}
$$

Then we define $\sum_{r=0}^{\infty} C_{r} \xi$ to be the analytic function $f(\xi)$ of $\xi$ in $I F$ if and only if $\xi(1)$ lies within $R$.

Write $f_{0}(x)=f(x), f_{a}(x)=d^{a} f(x) / d x^{a}(a>0), p=\xi(1)$. Then, by the above definition, if $f(\xi)$ is an analytic function of $\xi$ in $I F$; the series

$$
\sum_{j=0}^{\infty} c_{a+j} \frac{(a+j) !}{j !} p^{j}
$$

is absolutely convergent, and its sum is $f_{a}(p)(a \geqq 0)$.

Let $n$ be an arbitrary integer $>0$. Then (see $\$ 3$ ), the resolution of $\xi(n)$ contains at most $\pi(n)$ components $\xi_{a}(n)$. Denote $f(\xi)$ by $\Xi$. Then, if $\Xi$ is analytic in $I F$,

$$
\begin{aligned}
\Xi(n) & =\sum_{r=0}^{\infty} c_{r} \xi^{r}(n) \\
& =\sum_{r=0}^{\infty} r ! c_{r} \sum_{a=0}^{r} \frac{p^{r-a}}{(r-a) !} \xi_{a}(n) ;
\end{aligned}
$$

and the last is the sum of not more than $\pi(n)$ absolutely convergent series, 
the $\xi_{a}(n)$ being numerical constants with respect to the summations. Performing the summations, we have

$$
\Xi(n)=\sum_{a=0}^{\pi(n)} f_{a}(p) \xi_{a}(n) \quad(n=1,2, \cdots) ;
$$

or, what is the same, by the definition of equality in $I F$,

$$
f(\xi)=\sum_{a=0} f_{a}(p) \xi_{a}
$$

in which the upper limit of the summation may if desired be taken as $\infty$.

To summarize: If $\xi$ is any element of $I F$, and if $\xi(1)$ lies within the region of convergence of the scalar function $f(x)$, where

$$
f(x)=c_{0} x^{0}+c_{1} x+c_{2} x^{2}+\cdots,
$$

then, by definition, $f(\xi)$, where

$$
f(\xi)=c_{0} \eta+c_{1} \xi+c_{2} \xi^{2}+\cdots,
$$

is an analytic function of $\xi$ in $I F$, and it follows that

$$
f(\xi)=\sum_{a=0} f_{a}(p) \xi_{a} \quad(p=\xi(1)),
$$

where $f_{a}(p)$ is the scalar $\left[d^{a} f(x) / d x^{a}\right]_{x=p}$.

Denote by $f_{r}(x)(r \geqq 0)$ the $r$ th derivative of $f(x)$ with respect to $x$. Then, if $x_{0}$ lies within $R$, the series

$$
\sum_{j=0}^{\infty} c_{r+j} \frac{(r+j) !}{j !} x_{0}{ }^{i}
$$

is absolutely convergent and its sum is $f_{r}\left(x_{0}\right)$. Hence $f_{r}(\xi)$, where

$$
f_{r}(\xi)=\sum_{j=0}^{\infty} c_{r+j} \frac{(r+j) !}{j !} \xi^{i},
$$

is an analytic function in $I F$ of $\xi$, and, from what precedes and $\S 4$, we have

$$
f_{r}(\xi)=D_{\xi} f(\xi)=\sum_{a=0} f_{a}(p) \xi_{a-r},
$$

where $D_{\xi}^{r}$ indicates $r$ successive derivations with respect to $\xi$.

From (5.1), (5.2), we have the MacLaurin expansion of any analytic function $f(\xi)$ in $I F$,

$$
f(\xi)=\sum_{a=0}^{\infty} \frac{\xi^{a}}{a !} f_{a}(\omega)
$$


Similarly, if $\xi, \alpha$ are any elements of $I F$ such that $\xi(1)-\alpha(1)$ lies within the region of convergence of $f(x)$, we have the analogue in $I F$ of Taylor's theorem for scalars,

$$
f(\xi)=\sum_{a=0}^{\infty} \frac{(\xi-\alpha)^{a}}{a !} f_{a}(\alpha) .
$$

From the definition of analytic functions in $I F$, it is in short clear that the analysis of scalar analytic functions for values of their arguments within the complete intersection of the respective regions of convergence of the scalar functions goes over unchanged to IF by a mere reinterpretation of the scalar variabies as elements of $I F$ and of the four fundamental operations upon scalars as the corresponding operations in IF. (In division it is necessary to avoid irregular divisors.)

As a detail in the transposition from scalars to their unique correspondents in $I F$, we may repeat a remark in the introduction: The scalar unit 1 corresponds to $\eta$. For example, $\cos ^{2} \xi+\sin ^{2} \xi=\eta$ is true; $\cos ^{2} \xi+\sin ^{2} \xi=1$ ismeaningless, since $\cos ^{2} \xi, \sin ^{2} \xi$ are in $I F$, and 1 is not. Similarly for

$$
\sin \xi+\sin (-\xi)=\omega, \quad \sin \xi+\sin (-\xi)=0,
$$

the first of which is true and the second meaningless.

6 . The elementary functions in $I F$. In illustration of $\S 5$, we shall next briefly consider the exponential, circular and hyperbolic functions in $I F$. Since $p(=\xi(1))$ lies within the region of convergence of each of exp $x, \sin x$, $\cos x, \operatorname{sh} x, \operatorname{ch} x$ (because $\xi(1)$ is, by the definition of numerical functions, finite), each of $\exp \xi, \cdots, \operatorname{ch} \xi$, where $\xi$ is an arbitrary element of $I F$, is an analytic function of $\xi$ in $I F$. We have

$$
\begin{array}{rlrl}
\exp \xi & =\sum_{r=0}^{\infty} \frac{\xi^{r}}{r !} \\
\sin \xi & =\sum_{r=0}^{\infty} \frac{(-1)^{r} \xi^{2 r+1}}{(2 r+1) !}, & \cos \xi=\sum_{r=0}^{\infty} \frac{(-1)^{r} \xi^{2 r}}{(2 r) !} \\
\operatorname{sh} \xi & =\sum_{r=0}^{\infty} \frac{\xi^{2 r+1}}{(2 r+1) !}, & \operatorname{ch} \xi & =\sum_{r=0}^{\infty} \frac{\xi^{2 r}}{(2 r) !}
\end{array}
$$

Hence, if we define $E(\xi), \cdots, S(\xi)$ by

$$
\begin{aligned}
& E(\xi)=\sum_{a=0} \xi_{a}, \quad A(\xi)=\sum_{a=0} \xi_{2 a}, \quad B(\xi)=\sum_{a=0} \xi_{2 a+1}, \\
& C(\xi)=\sum_{a=0}\left(\xi_{4 a}-\xi_{4 a+2}\right), \quad S(\xi)=\sum_{a=0}\left(\xi_{4 a+1}-\xi_{4 a+3}\right),
\end{aligned}
$$


an immediate application of (5.1) gives the corresponding reduced forms,

$$
\begin{gathered}
\exp \xi=E(\xi) \exp p ; \\
\sin \xi=C(\xi) \sin p+S(\xi) \cos p, \\
\cos \xi=C(\xi) \cos p-S(\xi) \sin p ; \\
\operatorname{sh} \xi=A(\xi) \operatorname{ch} p+B(\xi) \operatorname{sh} p, \\
\operatorname{ch} \xi=A(\xi) \operatorname{sh} p+B(\xi) \operatorname{ch} p .
\end{gathered}
$$

From either (6.1) or (6.2) follow all the usual properties, except those concerning continuity and scalar periodicity, which are meaningless here, of scalar exponential, circular and hyperbolic functions for the corresponding analytic functions in $I F$ in exact isomorphism. Suppose these are developed. Then we may, at once, write down a second isomorphism, as follows:

\begin{tabular}{c|c|c|c|c}
$\exp x$ & $\sin x$ & $\cos x$ & $\operatorname{sh} x$ & $\operatorname{ch} x$ \\
\hline $\exp \xi$ & $\sin \xi$ & $\cos \xi$ & $\operatorname{sh} \xi$ & $\operatorname{ch} \xi$
\end{tabular},

corresponding functions being in the same column of the table, the complete isomorphism being such that, if any given relation holds between the members of a particular row of the table, say the first, as it is the most familiar, then the relation holds also when the members of that row are replaced by their correspondents in each of the remaining rows. The isomorphism includes derivatives, $D_{\xi}$ being the correspondent of $d / d x$.

In the preceding theory, we started from the fundamental theorem of arithmetic (unique decomposition into primes). There is a generalization to functions of the elements in any commutative semi-group, as may be seen from the algebra developed by the author.* The ultimate interpretation of properties of analytic functions is, as I will show elsewhere, a theory of compound partitions of matrices of rational integers.

* E. T. Bell, Algebraic Arithmetic, chapter IV.

California Institute of Technology, Pasadena, Calir. 